\title{
X-Ray Pulsar Profile Recovery Based on Tracking-Differentiator
}

\author{
Dapeng Zhang, Wei Zheng, Yidi Wang, and Lu Zhang \\ College of Aerospace Science and Engineering, National University of Defense Technology, Changsha 410073, China
}

Correspondence should be addressed to Wei Zheng; zhengwei@nudt.edu.cn

Received 2 February 2016; Revised 7 May 2016; Accepted 9 May 2016

Academic Editor: Yuming Qin

Copyright (c) 2016 Dapeng Zhang et al. This is an open access article distributed under the Creative Commons Attribution License, which permits unrestricted use, distribution, and reproduction in any medium, provided the original work is properly cited.

\begin{abstract}
The profile recovery is an important work in X-ray pulsar-based navigation. It is a key step for the analysis on the pulsar signal's characteristic and the computing of time of arrival (TOA). This paper makes an argument for an algorithm based on the trackingdifferentiator (TD) to recover the profile from the low Signal-to-Noise Ratio (SNR) signals. In the method, a TD filter with cascade structure is designed which has very low phase delay and amplitude distortion. In the simulation experiment, two typical pulsars (PSR B0531+21 and PSR B1937+21) are used to verify the algorithm's performance. The simulation results show that the method satisfies the application requirements in the aspects of SNR and profile fidelity. By processing the data collected by the Rossi X-Ray Timing Explorer (RXTE) satellite in space, similar results can also be achieved.
\end{abstract}

\section{Introduction}

A pulsar is a kind of neutron star rotating rapidly in space. An intersection angle exists between the pulsars' rotation axis and magnetic axis. The magnetic poles of the pulsars radiate $\mathrm{X}$-ray beam. When the X-ray beam sweeps on the measurement device, the device can receive the pulsar's signal [1]. Since the discovery of the first pulsar, scientists gradually recognized that the pulsar can be used as a natural clock because of its long-term stability. The X-ray pulsar-based navigation was initiated in the 1970s, and since then a complete navigation framework has already been developed. Since 1993, the United States carried out a series of research projects on X-ray pulsar-based navigation [2-5]. The European Space Agency (ESA) studied on the feasibility of X-ray pulsar-based navigation in 2004 [6]. Chinese scientists also began their research in 2004. In the aspects of signal processing and navigation algorithms, many valuable results have been achieved [7-9].

According to the distinctions of the energy bands of the pulsars, the pulsars can be divided into X-ray pulsars, radio pulsars, gamma-ray pulsars, and so forth. In order to reduce the mass and power dissipation of sensors, X-ray pulsars are mostly selected as navigation pulsars. Objectively, the signal of the X-ray pulsars is extremely weak so that only discrete photons can be recorded [10,11]. The background noise of the pulsar signals includes space background noise, high-energy particle noise, nebula noise, and detector noise. These noises can influence the precision of the pulsar profiles and increase navigation errors. Particularly, for the millisecond pulsar (e.g., PSR B1937+21), its signal is almost flooded in the noise. Figure 1 showed the influences caused by noise. Literature indicates that these noises can be modeled as Gauss white noise, and the variance is proportional to the number of the bins of the folding signal [12]. However, to get higherprecision profile needs to select more bins. One alternative method is to execute long-time pulsar observation. Nonetheless, owing to the limitation of the space environments and the task scheme of the aircraft, long-time observation is hard to implement. Therefore, it is quite necessary to research the profile recovery method in a shorter observation time.

The discrete tracking-differentiator (TD) is based on the second-order minimal time system. Since being proposed by Professor Jingqing $[13,14]$, the method has been applied in active disturbance rejection controllers (ADRC). Based on it, Xie and Long proposed a tracking-differentiator with a higher precision [15]. It can solve the disturbance problem of the signals effectively, track the input signal without overshooting, and obtain high-quality differential signal [16]. The structures of these two kinds of tracking-differentiators are different. Han's method is based on the transform of TD's nonlinear border and reachable region. Xie and Long's method is based on the switching curve and the time of reaching original point. In theory, the criterion of Xie and Long's method is stricter and faster. 


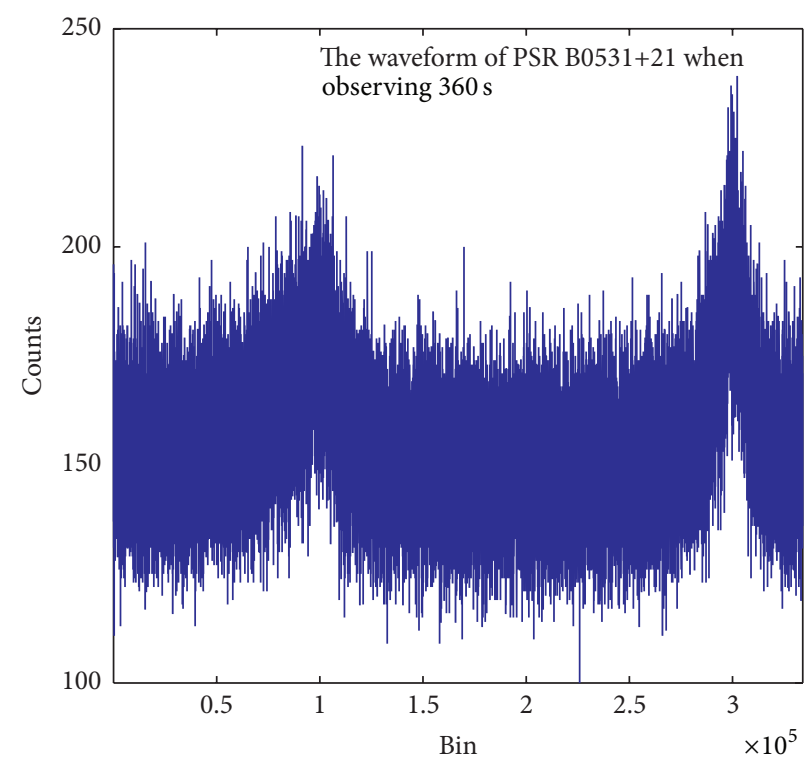

(a)

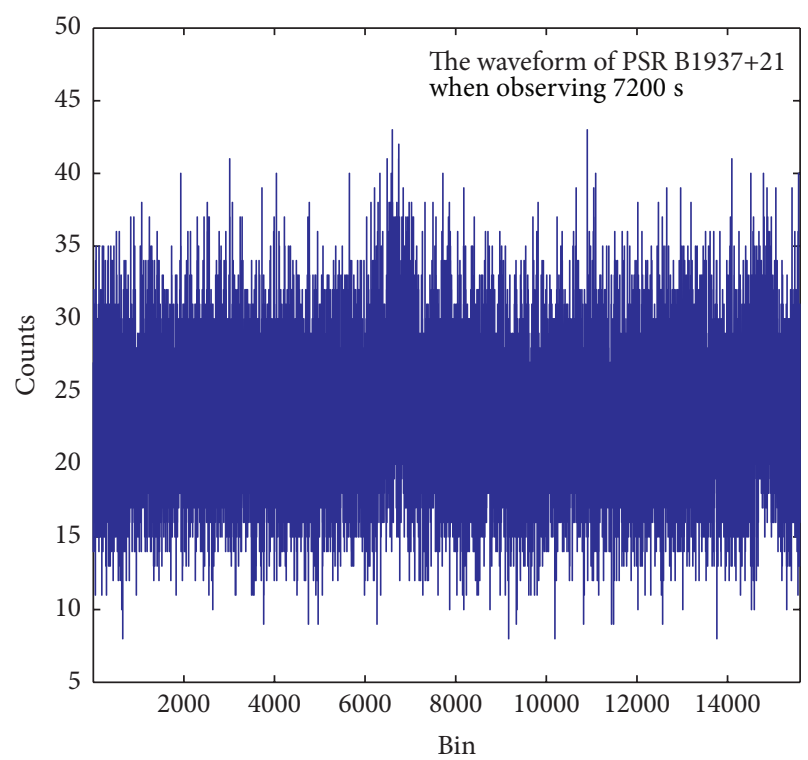

(b)

FIgURE 1: (a) The profile of PSR B0531+21 when observing $360 \mathrm{~s}$. (b) The profile of PSR B1937+21 when observing $720 \mathrm{s.}$
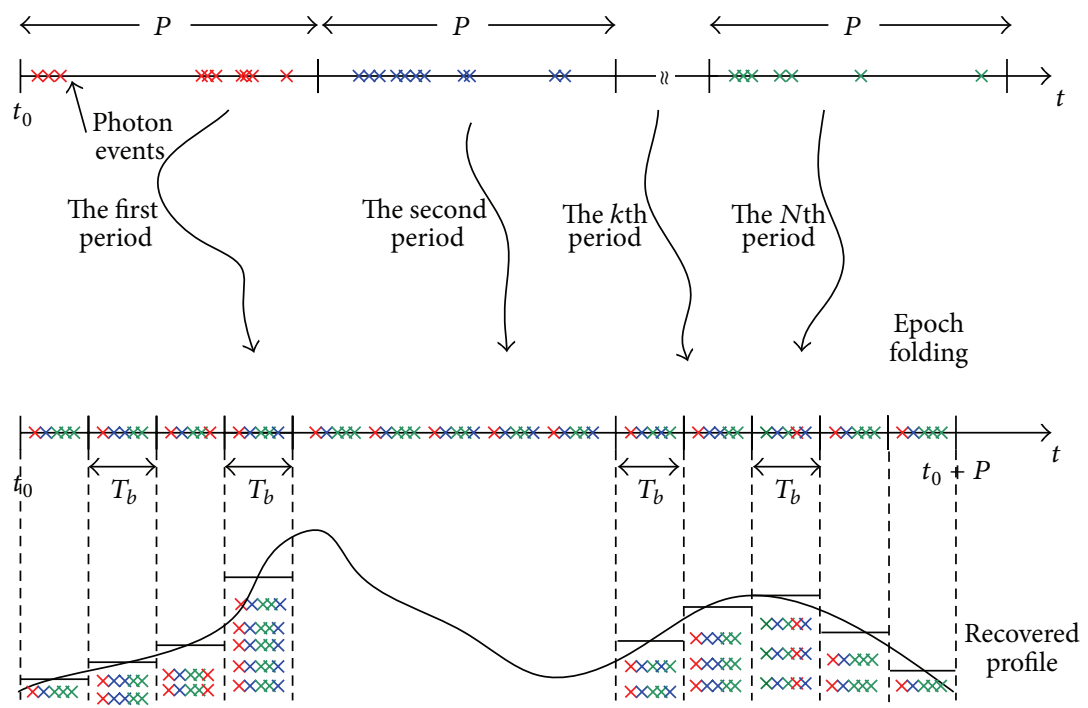

FIGURE 2: The principle of direct epoch folding.

\section{X-Ray Pulsar Profile Recovery}

2.1. The Epoch Folding of Discrete Photon Signal of Pulsar. In order to analyze the characteristics of pulsar signal and compute the TOA, the signal profile should be recovered first. At present, the method we usually use is direct epoch folding. The method includes 4 steps. (1) Perform barycenter correction. (2) Uniform the collected TOA photon series to a single pulse period. (3) Divide the period into some equal bins, and put the uniformed photons into their bins. (4) Compute the number of the photons in every bin, and plot the profile of the pulse. The process of direct epoch folding is shown in Figure 2.

\subsection{Profile Recovery Method Based on the Track-Differentiator}

2.2.1. The Algorithm of the Track-Differentiator. The discrete form of the track-differentiator can be expressed as follows:

$$
\begin{aligned}
{\left[\begin{array}{l}
x_{1}(k+1) \\
x_{2}(k+1)
\end{array}\right]=} & {\left[\begin{array}{ll}
1 & h \\
0 & 1
\end{array}\right]\left[\begin{array}{l}
x_{1}(k) \\
x_{2}(k)
\end{array}\right]+\left[\begin{array}{l}
\frac{h^{2}}{2} \\
h
\end{array}\right] u(k) } \\
& -\left[\begin{array}{c}
\frac{h^{3}}{3} \\
\frac{h^{2}}{2}
\end{array}\right] b(k),
\end{aligned}
$$




$$
|u(k)| \leq r
$$

$$
k=0,1,2, \ldots
$$

where $h$ is the step of sampling, $r$ is the factor of tracking, and $b(k)$ is the correction term. $h$ is selected according to time interval of data collection. $r$ decides the tracking speed and $b(k)$ can be calculated as (9).

If arbitrary point $M\left(x_{1}, x_{2}\right)$ in the phase plane $\Sigma=R^{2}$ is not on the switching curve $\Gamma\left(x_{1}, x_{2}\right)=x_{1}+x_{2}\left|x_{2}\right| / 2 r$, the time it takes to reach the switching curve is

$$
T_{A}=\frac{x_{2} s}{r}+\sqrt{\left|\frac{x_{1} s}{r}+\frac{1}{2} \frac{x_{2}^{2}}{r^{2}}\right|} .
$$

If $M\left(x_{1}, x_{2}\right)$ is on the switching curve, the time it takes to reach the original point is

$$
T_{B}=\frac{\left|x_{2}\right|}{r} .
$$

When $M\left(x_{1}, x_{2}\right)$ is not on the switching curve and cannot reach the switching curve in one step, the control law is

$$
u=-r \cdot \operatorname{sign}\left(\Gamma\left(x_{1}, x_{2}\right)\right) \text {. }
$$

Otherwise, the states $x_{1}$ and $x_{2}$ will be controlled to switching point in one step:

$$
u=-r \cdot s \cdot u_{a}
$$

where

$$
\begin{aligned}
u_{a} & =-\frac{1}{2}+\frac{x_{2} s}{r h}+\frac{1}{2} \sqrt{1+\frac{4}{r h}\left|x_{2}+\frac{2 x_{1}}{h}\right| s,} \\
s & =\operatorname{sign}\left(\Gamma\left(x_{1}, x_{2}\right)\right) .
\end{aligned}
$$

If $M\left(x_{1}, x_{2}\right)$ is on the switching curve $\Gamma$ and cannot reach the original point in one step, the control law is

$$
u=r \cdot \operatorname{sign}\left(x_{1}\right) \text {. }
$$

Otherwise,

$$
u=\frac{2}{h}\left(x_{2}+\frac{3 x_{1}}{h}\right)
$$

The correction term is given as follows:

$$
b=\frac{6}{h^{2}}\left(x_{2}+\frac{2 x_{1}}{h}\right) \text {. }
$$

\section{The Cascaded Filter Group}

The track-differentiator mentioned above can track lowfrequency signal quickly. In other words, it has a characteristic of low pass filter (TD filter), as Figure 3 shows. However, the signal processed by the single filter may lead to phase delay which cannot be accepted in the profile recovery application. Therefore, a filter group with cascaded structure

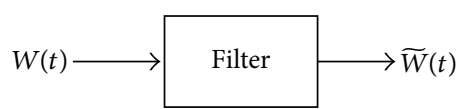

FIGURE 3: The IO model of the TD filter.

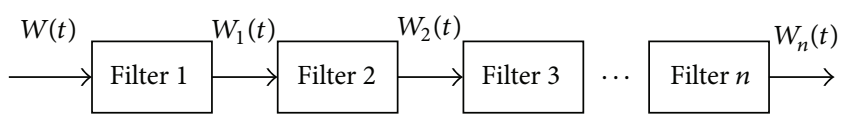

FIGURE 4: The cascaded filter group.

is designed below. The basic idea is to compensate the delay by multiple outputs of filters which has been applied in several engineering fields $[15,16]$.

The relation between the output and input signals is

$$
\widetilde{W}(t)=W(t-\tau)+\xi(t),
$$

where $\widetilde{W}(t)$ is output signal, $W(t)$ is input signal, and $\xi(t)$ is residual.

In order to compensate the delay $\tau$ in single filter, a filter group that consists of $n$ TD filters is established as Figure 4 shows.

The filter group can be expressed as

$$
\begin{aligned}
& W_{1}(t)=W(t-\tau)+\xi_{1}(t), \\
& W_{2}(t)=W(t-2 \tau)+\xi_{2}(t), \\
& W_{3}(t)=W(t-3 \tau)+\xi_{3}(t), \\
& W_{4}(t)=W(t-4 \tau)+\xi_{4}(t),
\end{aligned}
$$

$$
W_{n}(t)=W(t-n \tau)+\xi_{n}(t) .
$$

The Taylor expansion of $W(t-n \tau)$ is

$$
\begin{aligned}
W(t-i \tau)= & W(t)+\frac{d W(t)}{d t} \cdot(-i \tau)+\frac{d^{2} W(t)}{d t^{2}} \\
& \cdot \frac{(-i \tau)^{2}}{2 !}+\frac{d^{3} W(t)}{d t^{3}} \cdot \frac{(-i \tau)^{3}}{3 !}+R(t, \tau) .
\end{aligned}
$$

If we make $i=1,2,3,4$ (four-order Taylor expansion),

$$
\begin{aligned}
{\left[\begin{array}{l}
W_{1}(t) \\
W_{2}(t) \\
W_{3}(t) \\
W_{4}(t)
\end{array}\right]=} & {\left[\begin{array}{cccc}
1 & -\tau & \frac{\tau^{2}}{2} & -\frac{\tau^{3}}{6} \\
1 & -2 \tau & \frac{(2 \tau)^{2}}{2} & -\frac{(2 \tau)^{3}}{6} \\
1 & -3 \tau & \frac{(3 \tau)^{2}}{2} & -\frac{(3 \tau)^{3}}{6} \\
1 & -4 \tau & \frac{(4 \tau)^{2}}{2} & -\frac{(4 \tau)^{3}}{6}
\end{array}\right]\left[\begin{array}{c}
W(t) \\
W^{\prime}(t) \\
W^{\prime \prime}(t) \\
W^{\prime \prime \prime}(t)
\end{array}\right] } \\
& +\left[\begin{array}{l}
\xi_{1}(t) \\
\xi_{2}(t) \\
\xi_{3}(t) \\
\xi_{4}(t)
\end{array}\right] .
\end{aligned}
$$


Furthermore, separate the delay parameter $\tau$,

$$
\begin{aligned}
{\left[\begin{array}{l}
W_{1}(t) \\
W_{2}(t) \\
W_{3}(t) \\
W_{4}(t)
\end{array}\right]=} & {\left[\begin{array}{rrrr}
1 & -1 & \frac{1}{2} & -\frac{1}{6} \\
1 & -2 & 2 & -\frac{4}{3} \\
1 & -3 & \frac{9}{2} & -\frac{9}{2} \\
1 & -4 & 8 & -\frac{32}{3}
\end{array}\right]\left[\begin{array}{c}
W(t) \\
\tau W^{\prime}(t) \\
\tau^{2} W^{\prime \prime}(t) \\
\tau^{3} W^{\prime \prime \prime}(t)
\end{array}\right] } \\
& +\left[\begin{array}{l}
\xi_{1}(t) \\
\xi_{2}(t) \\
\xi_{3}(t) \\
\xi_{4}(t)
\end{array}\right] .
\end{aligned}
$$

Therefore,

$$
\begin{aligned}
{\left[\begin{array}{c}
W(t) \\
\tau W^{\prime}(t) \\
\tau^{2} W^{\prime \prime}(t) \\
\tau^{3} W^{\prime \prime \prime}(t)
\end{array}\right]=} & {\left[\begin{array}{cccc}
4 & -6 & 4 & -1 \\
4.3 & -9.5 & 7 & -1.8 \\
3 & -8 & 7 & -2 \\
1 & -3 & 3 & -1
\end{array}\right]\left[\begin{array}{l}
W_{1}(t) \\
W_{2}(t) \\
W_{3}(t) \\
W_{4}(t)
\end{array}\right] } \\
& -\left[\begin{array}{cccc}
4 & -6 & 4 & -1 \\
4.3 & -9.5 & 7 & -1.8 \\
3 & -8 & 7 & -2 \\
1 & -3 & 3 & -1
\end{array}\right]\left[\begin{array}{l}
\xi_{1}(t) \\
\xi_{2}(t) \\
\xi_{3}(t) \\
\xi_{4}(t)
\end{array}\right] .
\end{aligned}
$$

The input signal $W(t)$ can be denoted as

$$
W(t)=4 W_{1}(t)-6 W_{2}(t)+4 W_{3}(t)-W_{4}(t)-\Gamma(t),
$$

where $\Gamma(t)=4 \xi_{1}(t)-6 \xi_{2}(t)+4 \xi_{3}(t)-\xi_{4}(t)$ is residual noise of input signal.

Therefore, when the output signal is constructed to be

$$
\widetilde{W}(t)=4 W_{1}(t)-6 W_{2}(t)+4 W_{3}(t)-W_{4}(t)
$$

the output signal's phase is the same as the input signal. In other words, the phase delay is compensated. The structure of the filter group is as Figure 5 shows.

\section{Experiments}

4.1. Performance Analysis of the Filter Group. In general, the characteristics of amplitude-frequency and phase-frequency can better present the filters performance. Below, the designed filter group can be stimulated by the sinusoidal waves with different frequencies and the Bode diagrams were plotted.

Because the data processing here is off-line processing, the step of sampling $h$ can be arbitrarily set as 0.001 and the filtering factor $C_{0}$ can be set as 10,12 , or 15 . According to the experience equation $r=(2 \pi f \cdot A) /\left(h \cdot C_{0}\right)$, the tracking factor $r$ can be roughly set as 500 where the cut-off frequency $f$ is 4

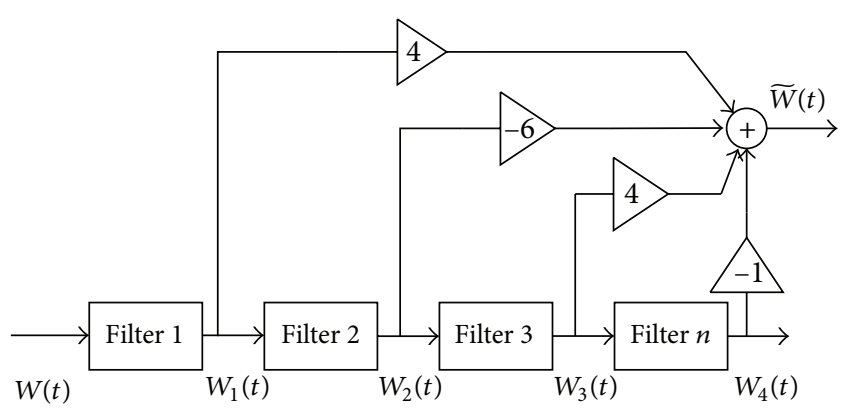

FIgURE 5: The structure of the filter group.

TABLE 1: Simulation parameters.

\begin{tabular}{lcc}
\hline Parameters & PSR B0531+21 & PSR B1937+21 \\
\hline Period (ms) & $33.4[17]$ & $1.56[4]$ \\
Flux (ph/s) & $660[18]$ & $0.5[4]$ \\
Flux of background noise (ph/s) & $13860[18]$ & $50^{\mathrm{a}}$ \\
Observation time (s) & 3600 & 7200 \\
Bins & 334000 & 15600 \\
\hline
\end{tabular}

${ }^{a}$ Estimated value. Cosmetic noise and detector noise are taken into consideration.

TABLE 2: Performance of the recovered profile.

\begin{tabular}{lcc}
\hline & PSR B0531+21 & PSR B1937+21 \\
\hline SNR $(\mathrm{dB})$ & 20.9 & 17.06 \\
$e_{\mathrm{r}}(\%)$ & 0.82 & 1.99 \\
$\Delta \phi($ cycle $)$ & $4.9 \times 10^{-5}$ & $1.34 \times 10^{-3}$ \\
\hline
\end{tabular}

and the gain $A$ is 1 . The order of Taylor expansion is 4,5 , or 6 . The results are shown in Figure 6.

From the results, it can be seen that the larger the filtering factor selected, the higher the cut-off frequency and the overshoot of the gain. Meanwhile, the variation trend of the signal phase is steeper. When the filter group with higher order is used, the overshoot is lower but the ability to suppress the high-frequency component is weaker. As shown in the amplitude-frequency and phase-frequency curve, the advantage of the TD filter group is the fact that, in the lowfrequency band, the processed signal has very low amplitude distortion and phase delay. The performance can almost get an ideal value.

4.2. Simulation Experiments of Pulsar Profile Recovery. In order to research the application range of the method the paper mentioned above, this section simulated two typical pulsars' data. The pulsars are PSR B0531+21 and PSR $\mathrm{B} 1937+21$. PSR B0531+21 is a young pulsar with very high radiation flux. PSR B1937+21 is an old pulsar with very low radiation flux. The parameters are shown in Table 1 . In this paper, two aspects are considered to measure the algorithm's performance. They are SNR (Signal-to-Noise Ratio) and fidelity. The fidelity is measured by two indices. 

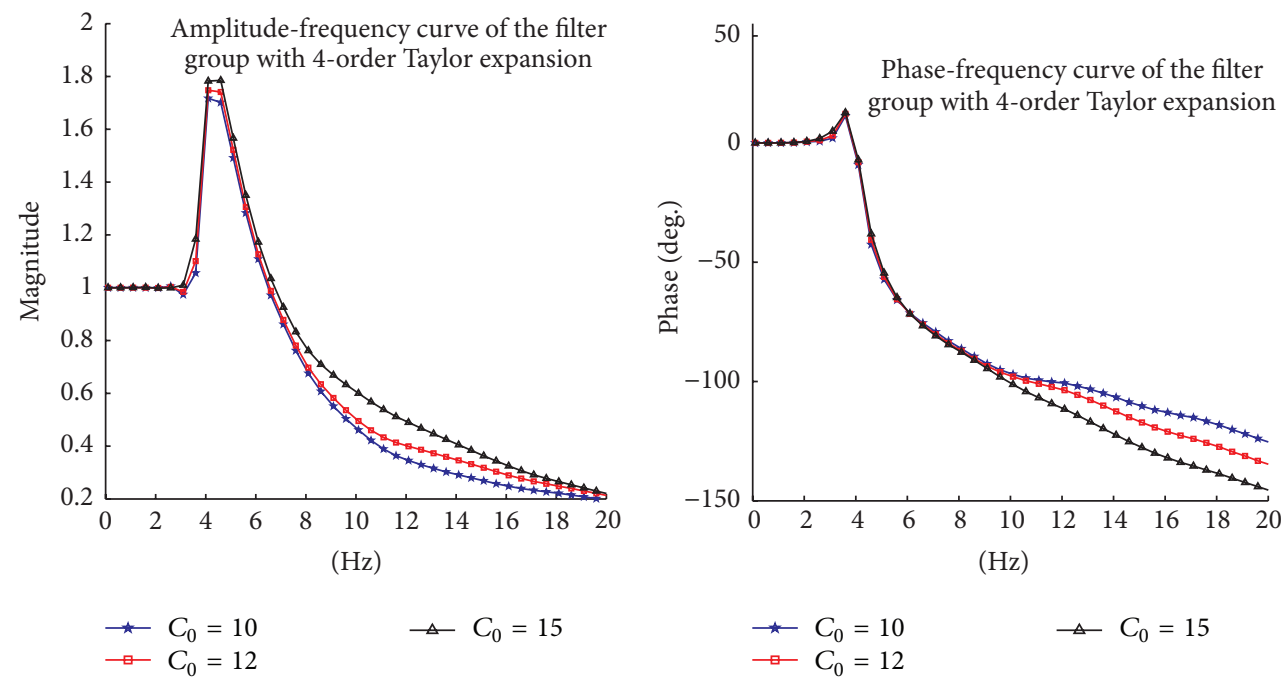

(a)

(b)
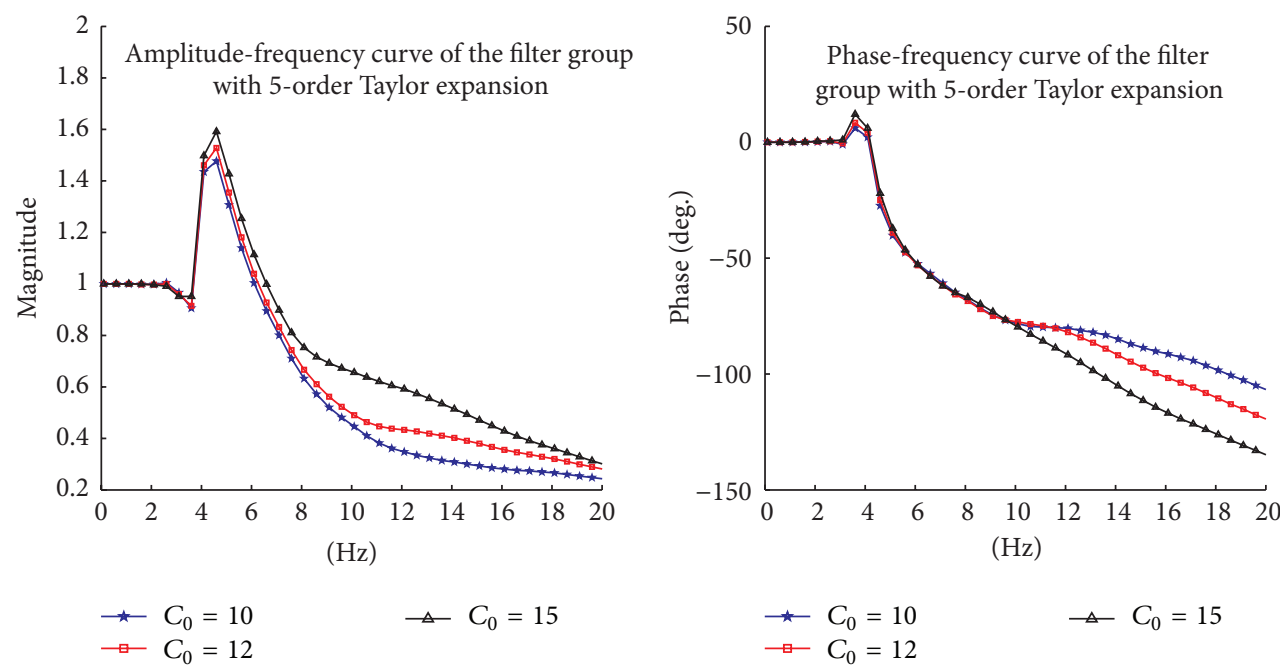

(c)

(d)

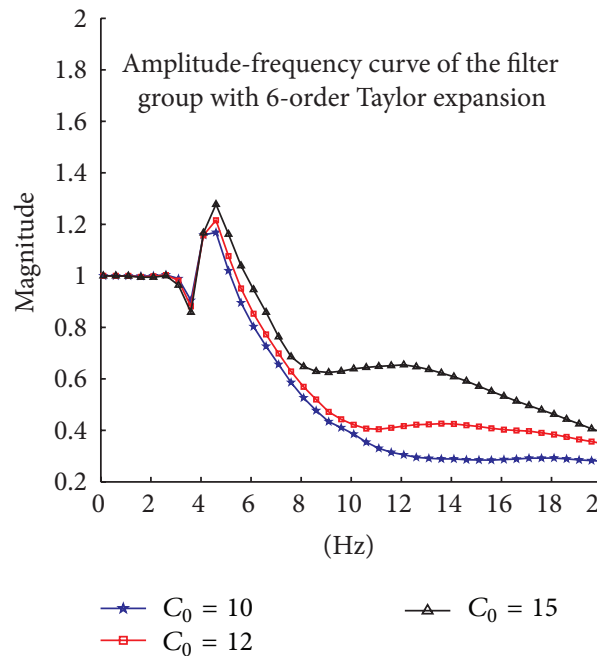

(e)

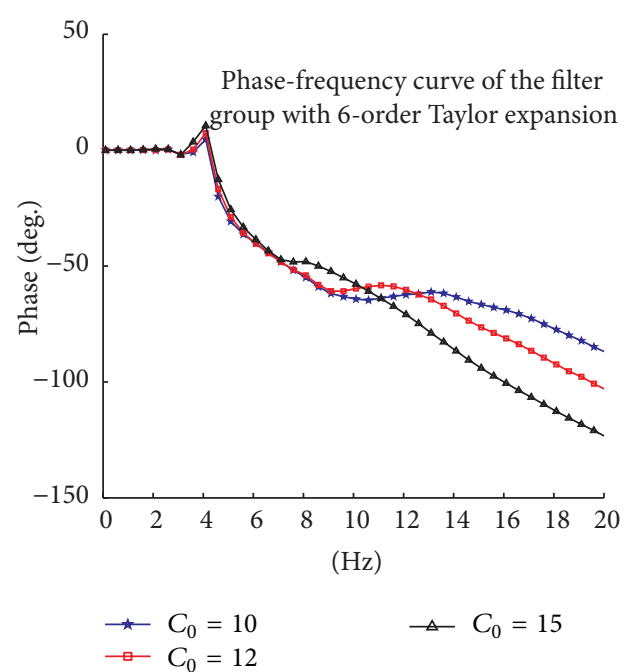

(f)

FIgure 6: (a) Amplitude-frequency curve of the filter group with 4-order Taylor expansion. (b) Phase-frequency curve of the filter group with 4-order Taylor expansion. (c) Amplitude-frequency curve of the filter group with 5-order Taylor expansion. (d) Phase-frequency curve of the filter group with 5-order Taylor expansion. (e) Amplitude-frequency curve of the filter group with 6-order Taylor expansion. (f) Phasefrequency curve of the filter group with 6-order Taylor expansion. 
TABle 3: Pulsar parameters [4].

\begin{tabular}{lccccc}
\hline Parameters & Period $(\mathrm{ms})$ & Flux $^{\mathrm{a}}\left(\mathrm{ph} / \mathrm{cm}^{2} / \mathrm{s}\right)$ & Distance $(\mathrm{kpc})$ & Galactic longitude (deg.) & Galactic latitude $(\mathrm{deg})$. \\
\hline PSR B0531+21 & 33.4 & 1.54 & 2 & 184.56 & -5.78 \\
PSR B1509-58 & 150.23 & $1.62 E-02$ & 4.3 & 320.32 & -1.16 \\
PSR B0540-69 & 50.37 & $5.15 E-03$ & 47.3 & 279.72 & -31.52 \\
\hline
\end{tabular}

${ }^{\text {a }}$ The energy range is $2-10 \mathrm{keV}$.

TABLE 4: Experiment data information.

\begin{tabular}{|c|c|c|c|c|c|}
\hline \multirow{2}{*}{ Parameters } & \multirow{2}{*}{ Observational ID } & \multirow{2}{*}{ Effective data length } & \multicolumn{3}{|c|}{ Discriminant conditions of effective data } \\
\hline & & & $\mathrm{elv}^{\mathrm{a}}$ & Offset $^{b}$ & num_pcu_on $^{c}$ \\
\hline \multirow{3}{*}{ PSR B0531+21 } & 20804-01-01-00 & $9921 \mathrm{~s}$ & $>10$ & $<0.01$ & $\geq 1$ \\
\hline & 40805-01-05-01 & $8789 \mathrm{~s}$ & $>10$ & $<0.01$ & $\geq 1$ \\
\hline & 50098-01-01-00 & $1829 \mathrm{~s}$ & $>10$ & $<0.01$ & $\geq 1$ \\
\hline PSR B1509-58 & 94803-01-11-06 & $10298 \mathrm{~s}$ & $>10$ & $<0.01$ & $\geq 1$ \\
\hline PSR B0540-69 & 10218-01-01-00 & $21816 \mathrm{~s}$ & $>10$ & $<0.01$ & $\geq 1$ \\
\hline
\end{tabular}

${ }^{a}$ elv means elevation angle. ${ }^{b}$ Offset means pointing position. ${ }^{c}$ num_pcu_on means the number of the working sensors.

\subsubsection{Relative Error of the Recovered Profile $e_{r}$. Consider}

$$
e_{\mathrm{r}}=\sqrt{\frac{\sum_{i=1}^{\mathrm{bin}}\left(\tilde{\lambda}\left(\phi_{i}\right)-\lambda\left(\phi_{i}\right)\right)^{2}}{\sum_{i=1}^{\mathrm{bin}} \lambda^{2}\left(\phi_{i}\right)}} \times 100 \%
$$

where $\tilde{\lambda}(\cdot)$ is the recovered profile, $\lambda(\cdot)$ is the standard profile, and bin is the number of the bins.

4.2.2. The Phase Bias of the Recovered Profile $\Delta \phi$. This value is computed by the method of cross-correlation between the recovered profile and standard profile. In this paper, the results are obtained by 1000 Monte Carlo simulations.

The parameters of the filter group are $r=800, h=0.001$, $C_{0}=50$, and the order of the Taylor expansion is 5 .

The performance of the recovered profile is shown in Table 2 and the result pictures are shown in Figure 7.

4.3. RXTE Data Experiments of Pulsar Profile Recovery. The RXTE (Rossi X-Ray Timing Explorer) satellite was launched by the USA in 1995. It has operated over 16 years and obtains a large amount of pulsar photon data $[19,20]$. The detector on RXTE has high time resolution and high sensitivity. The data observed by RXTE has supported a large number of scientific researches [21-24].

In order to verify the feasibility of the method, the data detected by RXTE is used in this section. We choose three pulsars to verify the method. They are PSR B0531+21, PSR B1509-58, and PSR B0540-69. The basic parameters are shown in Table 3. In this experiment we used PCA Event Encoded Mode data. The PSR B0521+21 data packages we used are "20804-01-01-00," "40805-01-05-01," and "50098-0101-00." The PSR B1509-58 data package is "94803-01-11-06" and PSR B0540-69 data package is "10218-01-01-00." Heasoft (v6.17) was used to process the observational data as follows. (1) Remove clock events with the "sefilter" command for PSR B0531+21 or combine FITS files with the "se_make" command for PSR B1509-58 and PSR B0540-69. (2) Create filter file with the "xtefilt" command. (3) Generate the Good Time Interval (GTI) file with the "maketime" command. (4) Create good events file with the "grosstimefilt" command. (5) Correct the barycenter with the "faxbary" command. Therefore the original experiment data is obtained. The data information is shown in Table 4. Taking advantage of the method of $\chi^{2}$ searching, the signal's period is computed and the algorithm is as the following equation shows:

$$
\begin{aligned}
\chi^{2} & =\sum_{i=1}^{m} \frac{\left(n_{i}-N / m\right)^{2}}{N / m}, \\
\widehat{P} & =\arg \left[\max \left(\chi^{2}\right)\right],
\end{aligned}
$$

where $m$ is the bin number of the profile and total photon number $N=\sum n_{i}$, where $n_{i}$ is the photon number in the $i$ th bin. And then, by epoch folding, the original profile waveform is obtained.

Use the profile recovery method mentioned above. The data processing results of PSR B0531+21 are shown in Figure 8.

The data processing results of PSR B1509-58 are shown in Figure 9 and of PSR B0540-69 in Figure 10.

Figures 8-10 show the recovered profile based on the tracking-differentiator. By comparing with the simulation results in Section 4.2, the results in this section are consistent with them. The method is proved to be effective.

\section{Conclusions}

In order to enhance the SNR of the recovered profiles of the pulsars, the paper introduces a profile recovery method based on tracking-differentiator. Applying the method, a cascaded structure filter group is constructed. The filter group reduces the phase distortion and enhances the performance of the recovered profile. By simulating the photon data of the two typical pulsars (PSR B0531+21 and PSR B1937+21), the paper 


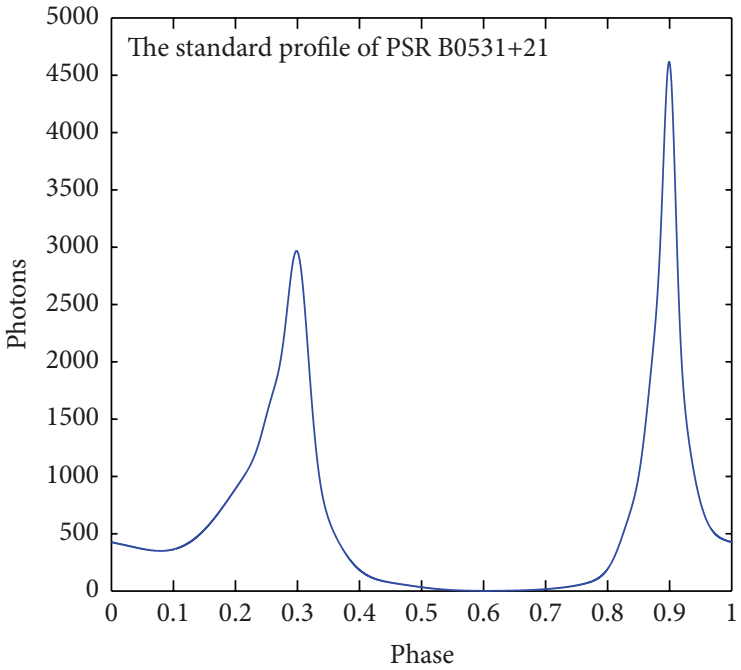

(a)

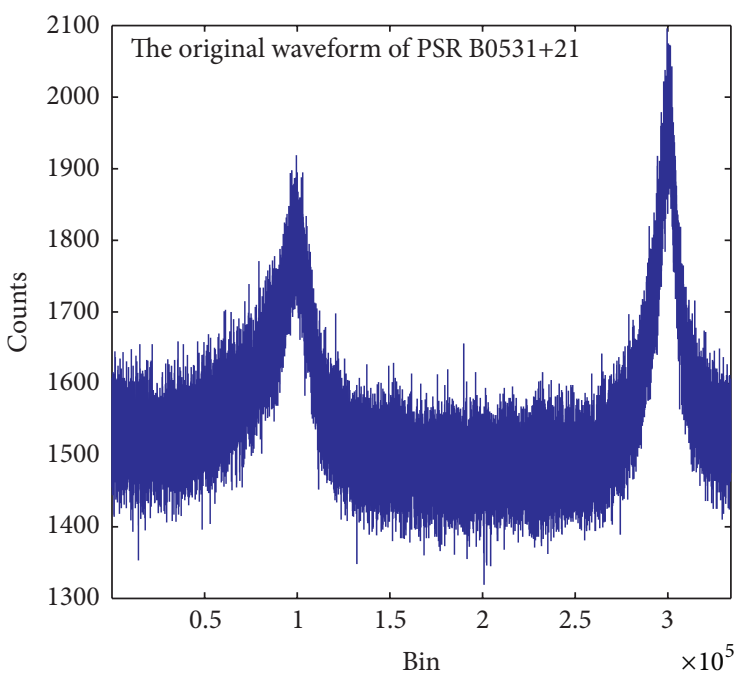

(c)

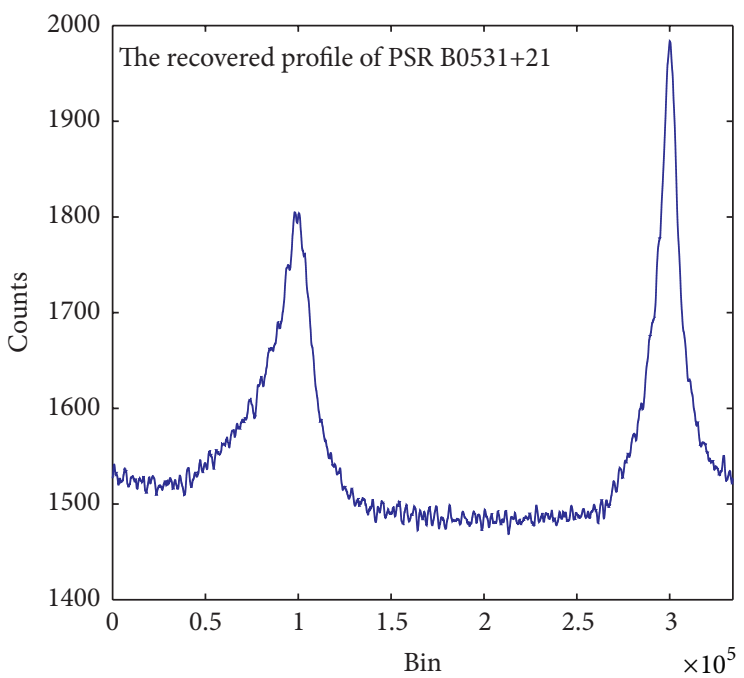

(e)

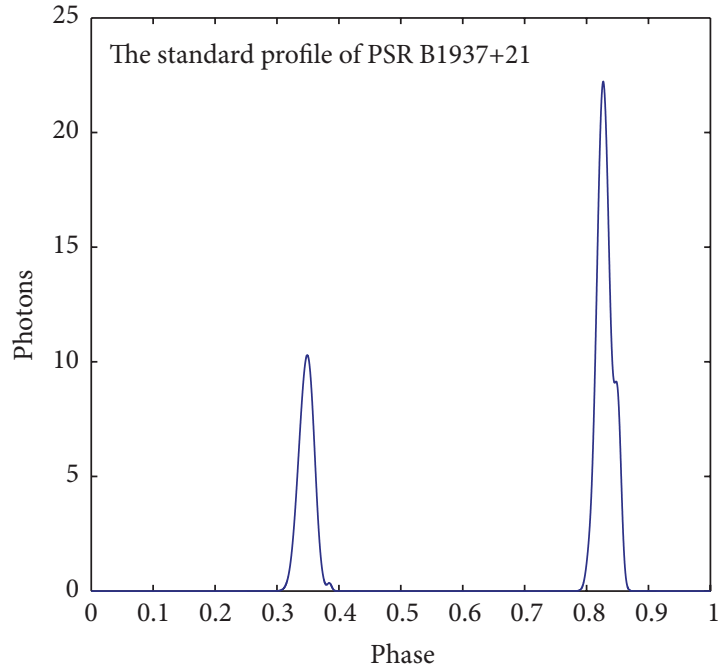

(b)

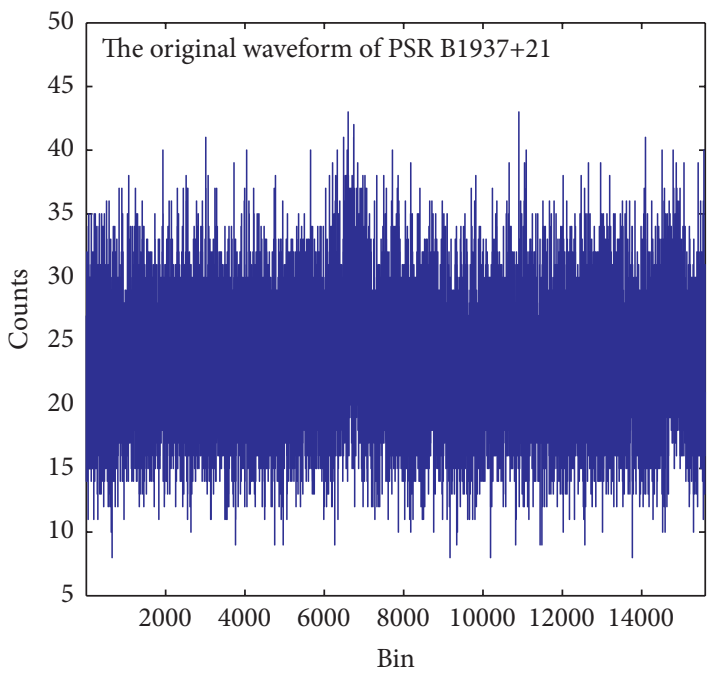

(d)

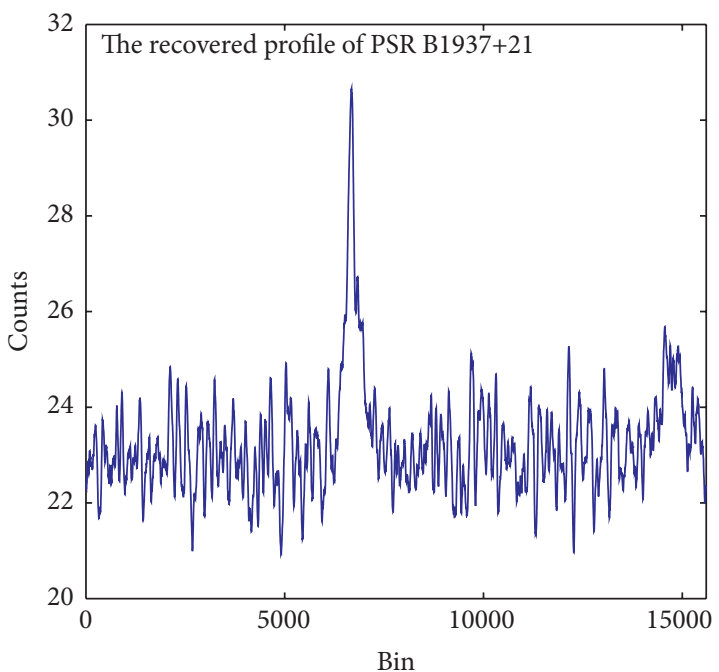

(f)

FIGURE 7: (a) The standard profile of PSR B0531+21. (b) The standard profile of PSR B1937+21. (c) The original wave of PSR B0531+21. (d) The original wave of PSR B1937+21. (e) The recovered profile of PSR B0531+21. (f) The recovered profile of PSR B1937+21. 


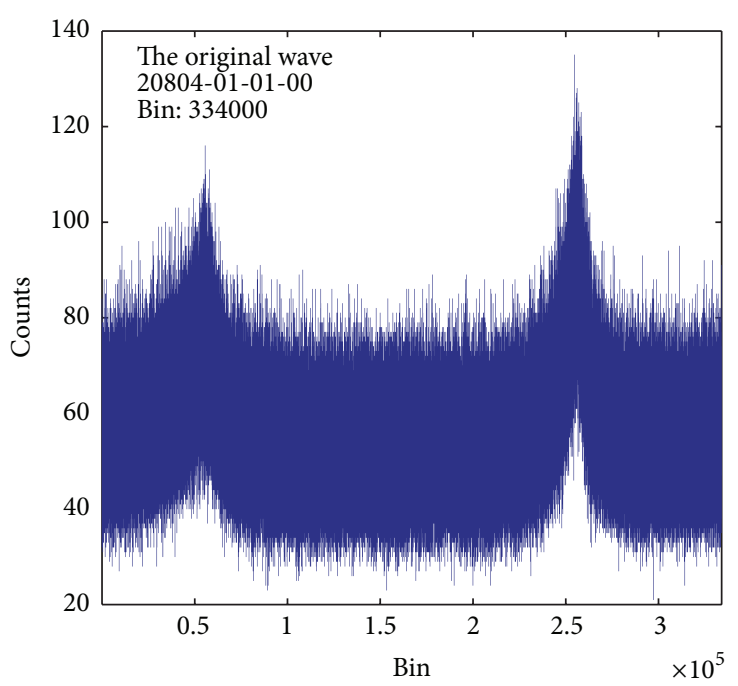

(a)

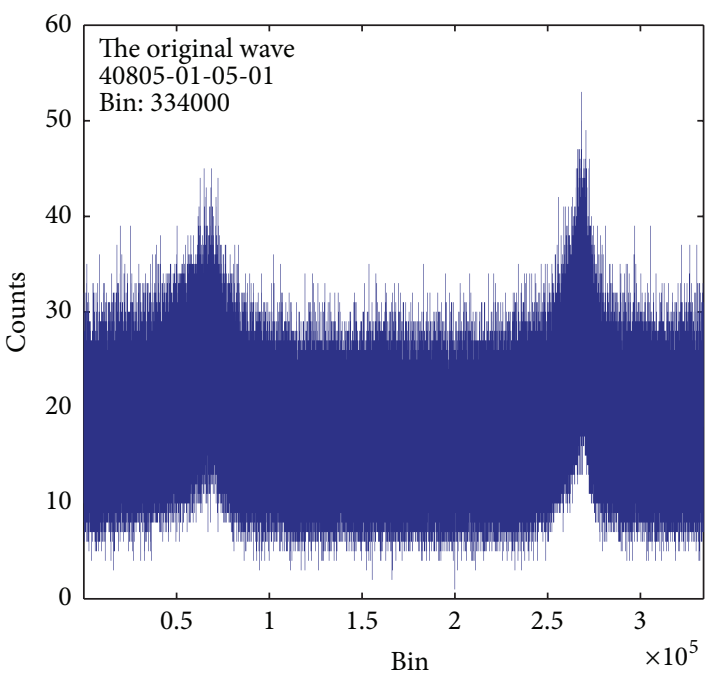

(c)

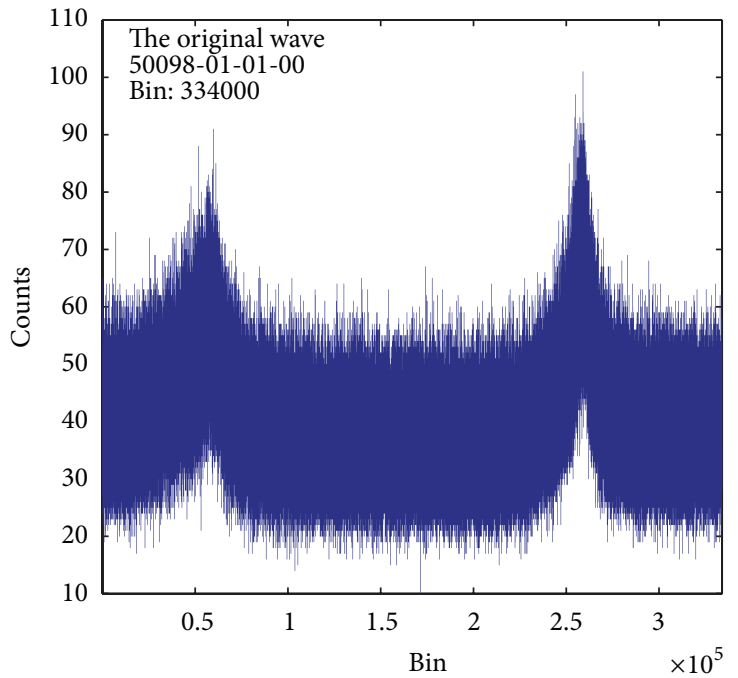

(e)

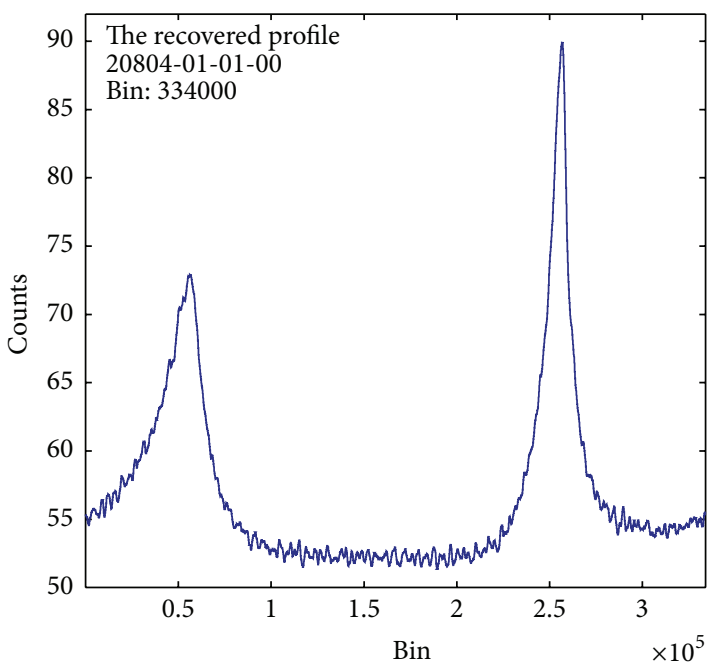

(b)

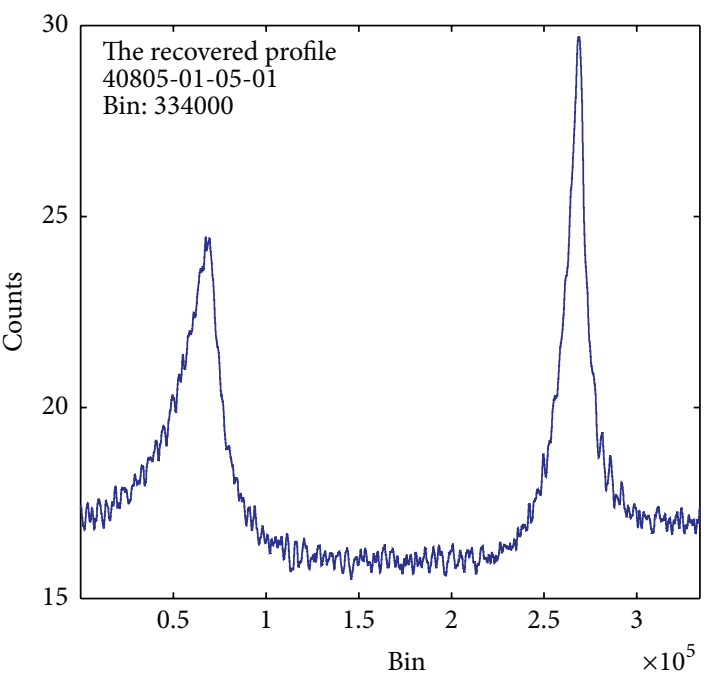

(d)

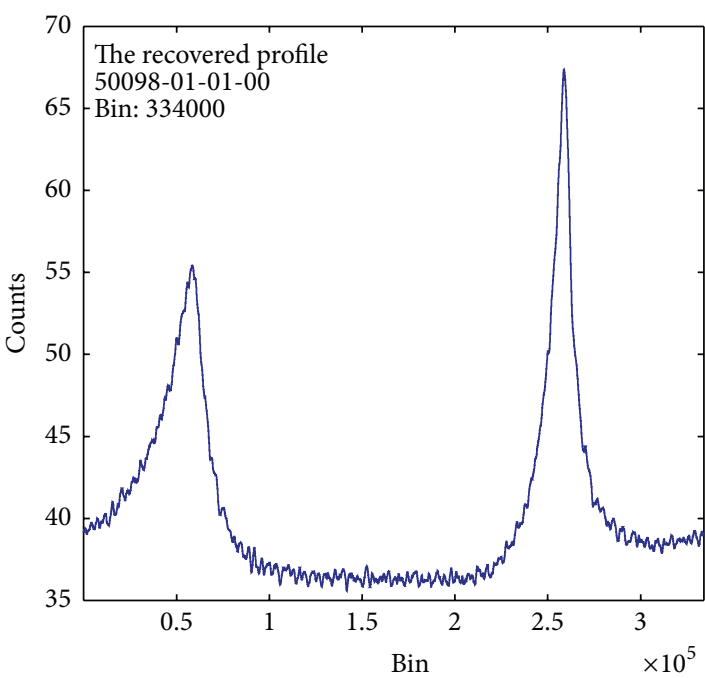

(f)

FIGURE 8: The experiment results of PSR B0531+21. 


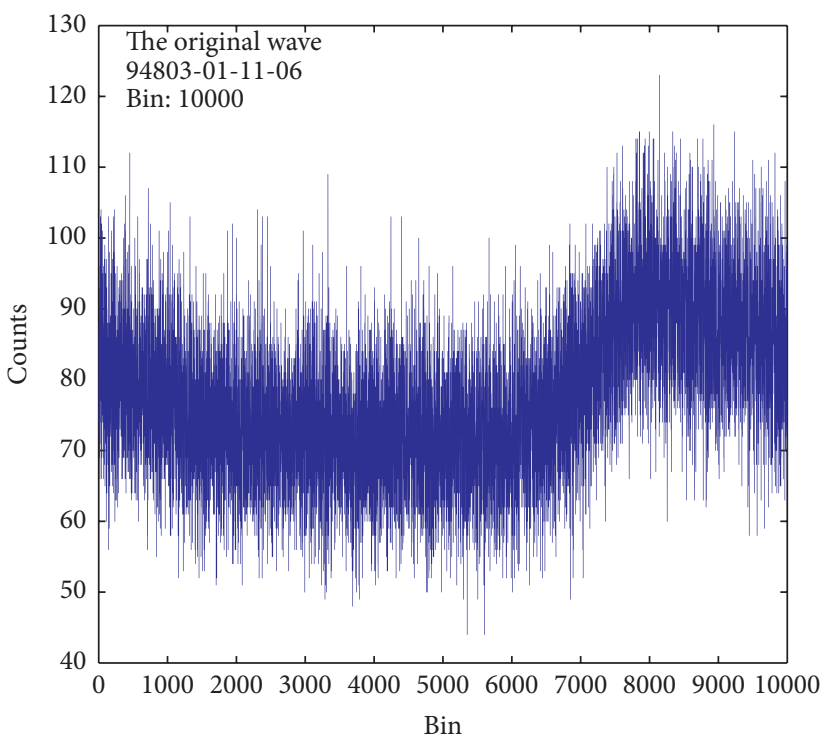

(a)

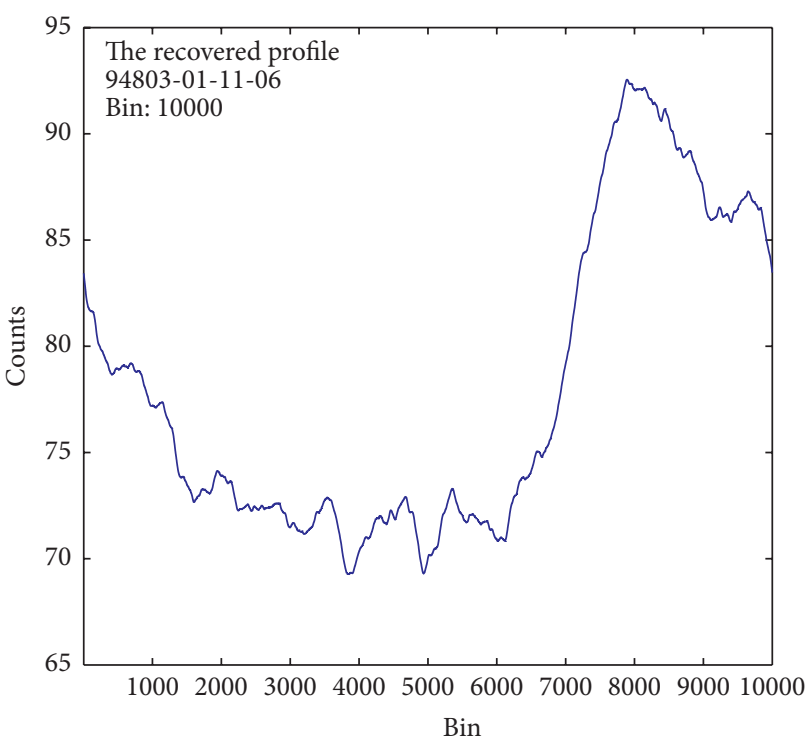

(b)

FIGURE 9: The experiment results of PSR B1509-58.

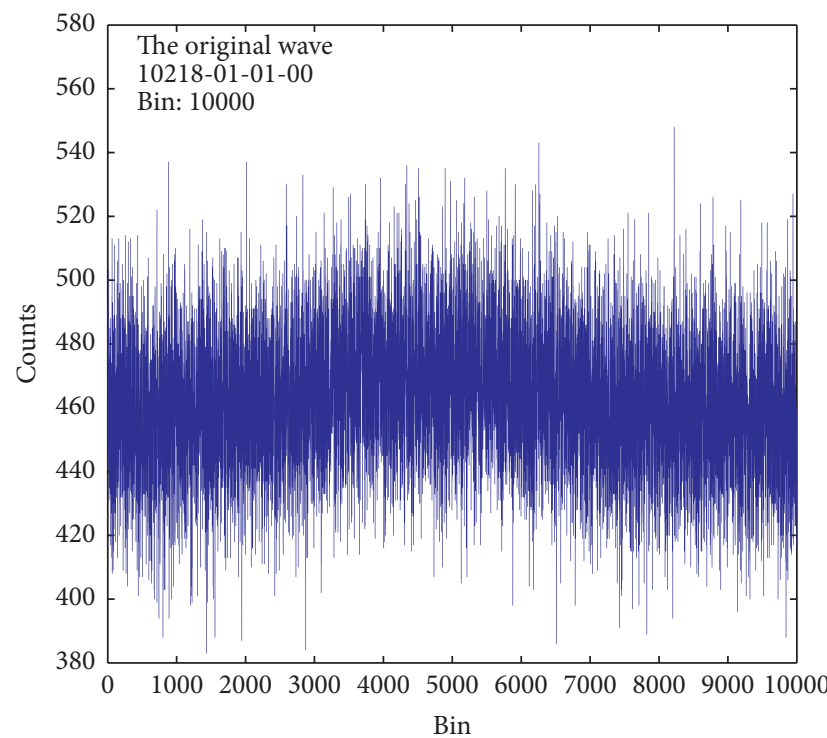

(a)

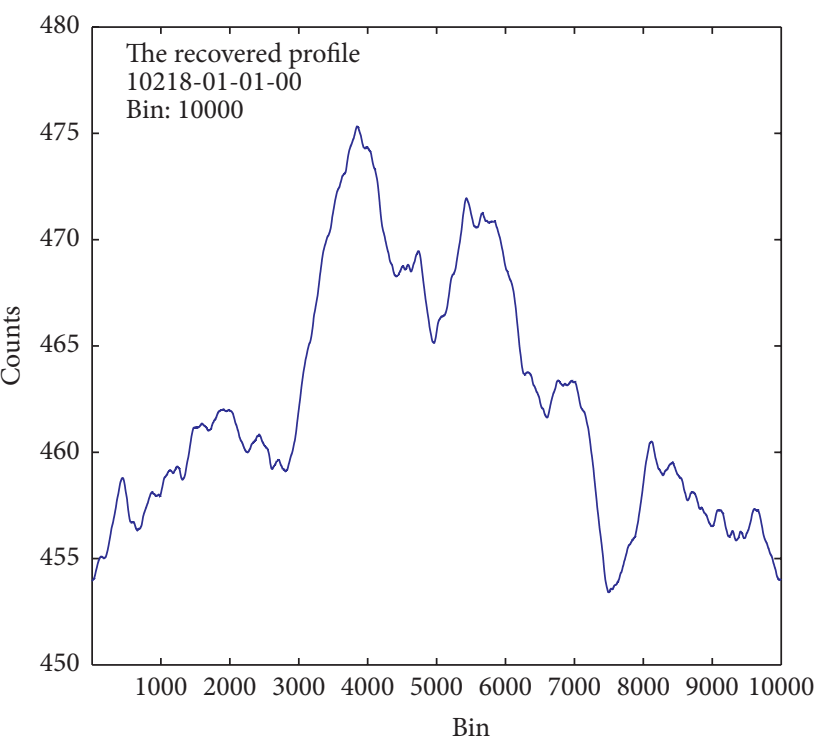

(b)

FIGURE 10: The experiment results of PSR B0540-69.

analyzes the performance of the method. The results show that the method can meet requirements in the aspects of the SNR and fidelity and is appropriate for both young pulsars and old pulsars. By processing the measured data from RXTE satellite, the simulation results are verified. The method does not need the a priori information of the pulsar standard profile, and it can be applied in the X-ray pulsar-based navigation fields.

\section{Competing Interests}

The authors declare that they have no competing interests.

\section{Acknowledgments}

The authors thank the National Aeronautics and Space Administration (NASA) for using its open data observed by the RXTE satellite.

\section{References}

[1] A. Lyne and F. Graham-Smith, Pulsar Astronomy, University Press, Cambridge, UK, 2012.

[2] J. Hanson, Principles of X-Ray Navigation, Stanford University, Stanford, Calif, USA, 1996. 
[3] T. C. John, Autonomous X-Ray Pulsar Based Spacecraft Navigation, Microcosm Inc, Hawthorne, Calif, USA, 2004.

[4] S. I. Sheikh, The use of variable celestial X-ray sources for spacecraft navigation [Ph.D. thesis], University of Maryland, 2005.

[5] J. W. Mitchell, M. Hassouneh, L. Winternitz et al., "SEXTANTstation explorer for X-ray timing and navigation technology," in Proceedings of the AIAA Guidance, Navigation, and Control Conference, Kissimmee, Fla, USA, 2015.

[6] S. Alvarez, J. Planas, A. Piera et al., Pulsar Navigation, European Space Agency, 2008.

[7] W. Zheng, Y. Wang, G. Tang, and L. Liu, X-Ray Pulsar-Based Navigation: Theory and Applications, Science Press, Beijing, China, 2015.

[8] Y. D. Wang and W. Zheng, "Pulse phase estimation of X-ray pulsar with the aid of vehicle orbital dynamics," Journal of Navigation, vol. 69, no. 2, pp. 414-432, 2016.

[9] Y. D. Wang, W. Zheng, S. M. Sun, and L. Li, "X-ray pulsar-based navigation system with the errors in the planetary ephemerides for Earth-orbiting satellite," Advances in Space Research, vol. 51, no. 12, pp. 2394-2404, 2013.

[10] H.-F. Sun, W.-M. Bao, H.-Y. Fang, and X.-P. Li, "Effect of stability of X-ray pulsar profiles on range measurement accuracy in X-ray pulsar navigation," Acta Physica Sinica, vol. 63, no. 6, Article ID 069701, 2014.

[11] P. Pradhan, B. Paul, H. Raichur, and B. C. Paul, "Variations of the harmonic components of the X-ray pulse profile of PSR B150958," Research in Astronomy and Astrophysics, vol. 15, no. 1, pp. 28-36, 2015.

[12] A. Emadzadeh and J. Speyer, Navigation in Space by X-Ray Pulsars, Spring Press, London, UK, 2011.

[13] J. Han and W. Wang, "Nonlinear tracking-differentiator," Journal of Systems Science \& Complexity, vol. 2, pp. 177-180, 1994.

[14] H. Jingqing, Active Disturbance Rejection Control TechnologyThe Technique for Estimating and Compensating the Uncertainties, National Defense Industry Press, 2009.

[15] Y.-D. Xie and Z.-Q. Long, "High-speed nonlinear discrete tracking-differentiator with high precision," Control Theory \& Applications, vol. 26, no. 2, pp. 127-132, 2009.

[16] C. Dai, Z. Long, Y. Xie, and S. Xue, "Research on the filtering algorithm in speed and position detection of maglev trains," Sensors, vol. 11, no. 7, pp. 7204-7218, 2011.

[17] Y. D. Wang, W. Zheng, S. M. Sun, and L. Li, "X-ray pulsar-based navigation using time-differenced measurement," Aerospace Science and Technology, vol. 36, pp. 27-35, 2014.

[18] L. M. B. Winternitz, M. A. Hassouneh, J. W. Mitchell et al., "Xray pulsar navigation algorithms and testbed for SEXTANT," in Proceedings of the IEEE Aerospace Conference (AERO '15), pp. 1-14, IEEE, Big Sky, Mont, USA, March 2015.

[19] O. W. Butters, A. J. Norton, K. Mukai, and J. A. Tomsick, "RXTE and XMM observations of intermediate polar candidates," Astronomy \& Astrophysics, vol. 526, no. 9, article A77, 2011.

[20] M. Y. Ge, F. J. Lu, J. L. Qu, S. J. Zheng, Y. Chen, and D. W. Han, "X-ray phase-resolved spectroscopy of PSRs B0531+21, B1509-58, and B0540-69 with RXTE," Astrophysical Journal, Supplement Series, vol. 199, pp. 8-10, 2012.

[21] C. B. Markwardt and J. H. Swank, RXTE PCA Detects the Millisecond Pulsar SAX J1808.4-3658 in Outburst, vol. 1728 of Astromomers Telegram, 2008.

[22] M. Vivekanand, "RXTE observations of single pulses of PSR B0531+21 II. Test for radio behavior," Astronomy and Astrophysics, vol. 376, no. 2, pp. 580-585, 2001.
[23] G. Cusumano, W. Hermsen, M. Kramer et al., "The phase of the radio and X-ray pulses of PSR B1937+21," Nuclear Physics BProceedings Supplements, vol. 132, pp. 596-599, 2004.

[24] Y. Saito, N. Kawai, A. Rots et al., "RXTE observation of the millisecond pulsar PSR B1821-24," in New Century of XRay Astronomy, ASP Conference Proceedings, vol. 251, pp. 1-6, Astronomical Society of the Pacific, 2001. 


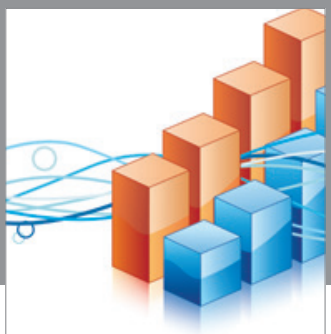

Advances in

Operations Research

vatem alat4

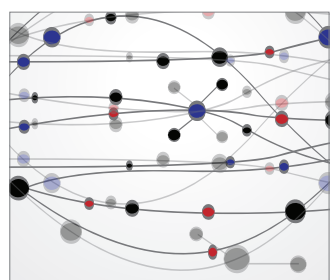

\section{The Scientific} World Journal
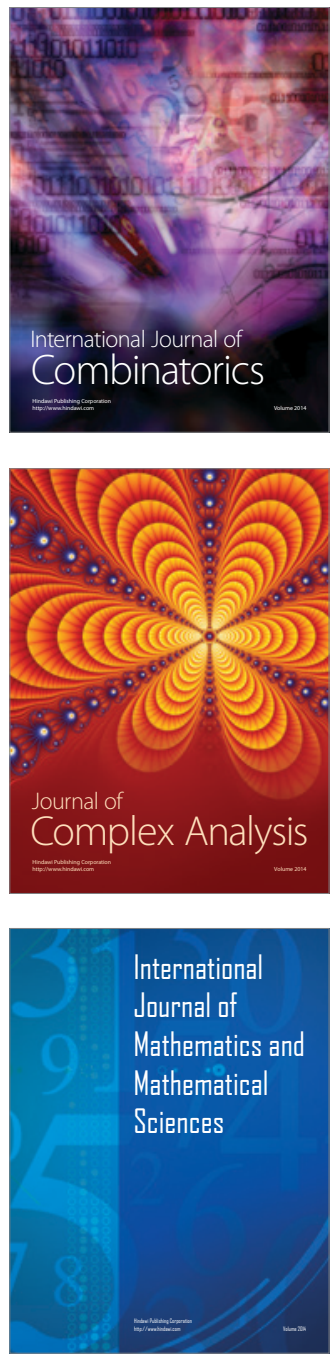
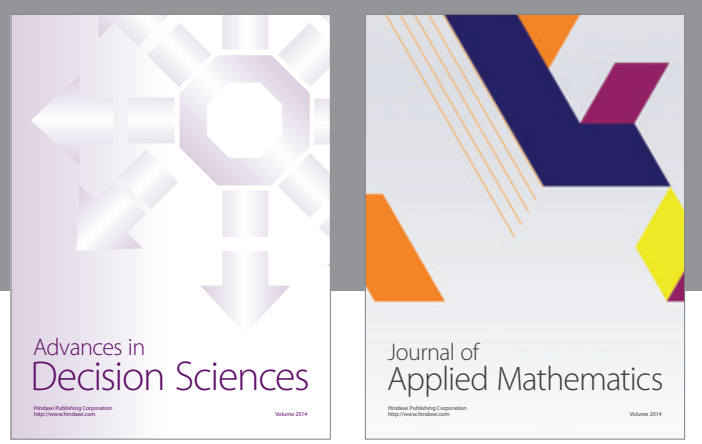

Algebra

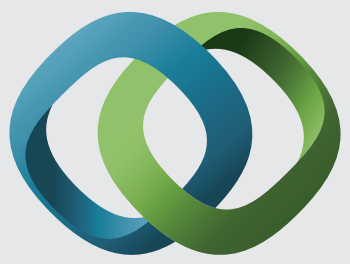

\section{Hindawi}

Submit your manuscripts at

http://www.hindawi.com
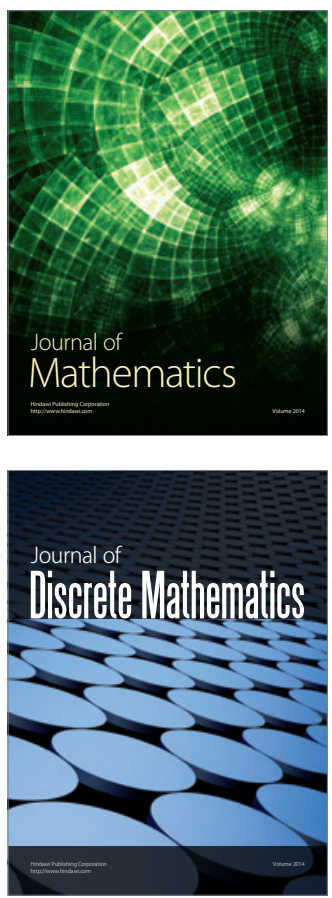

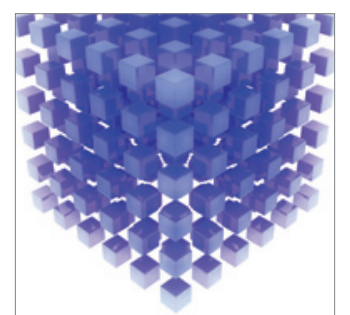

Mathematical Problems in Engineering
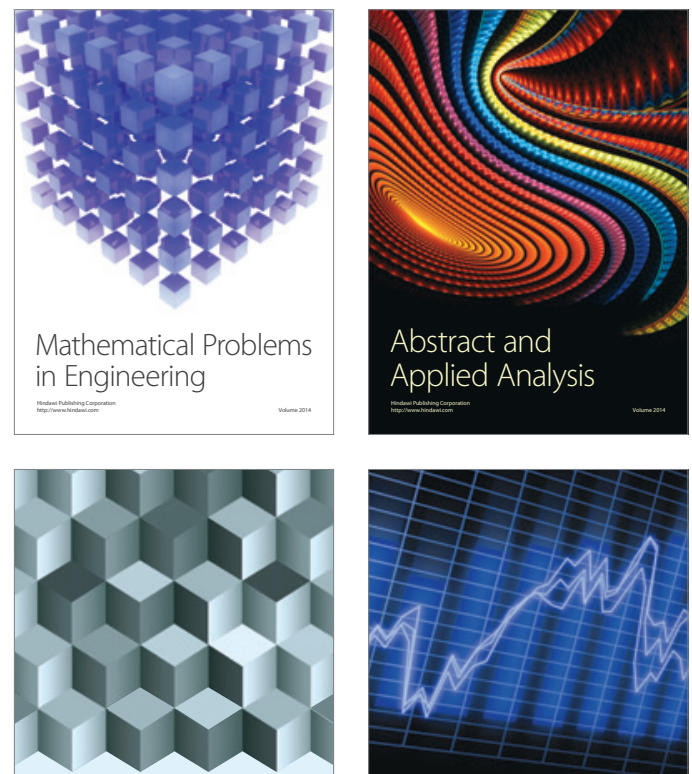

Journal of

Function Spaces

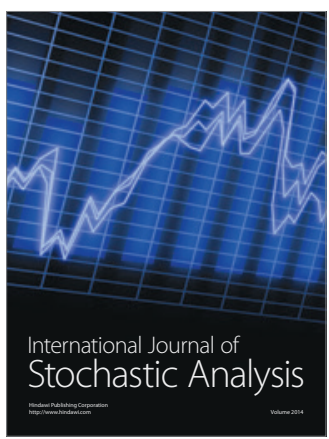

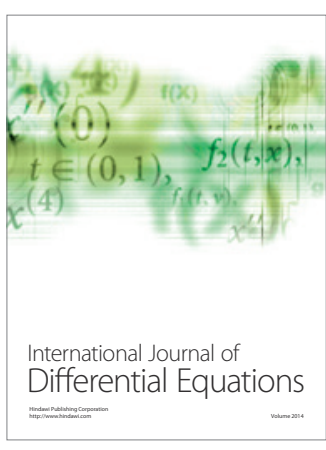
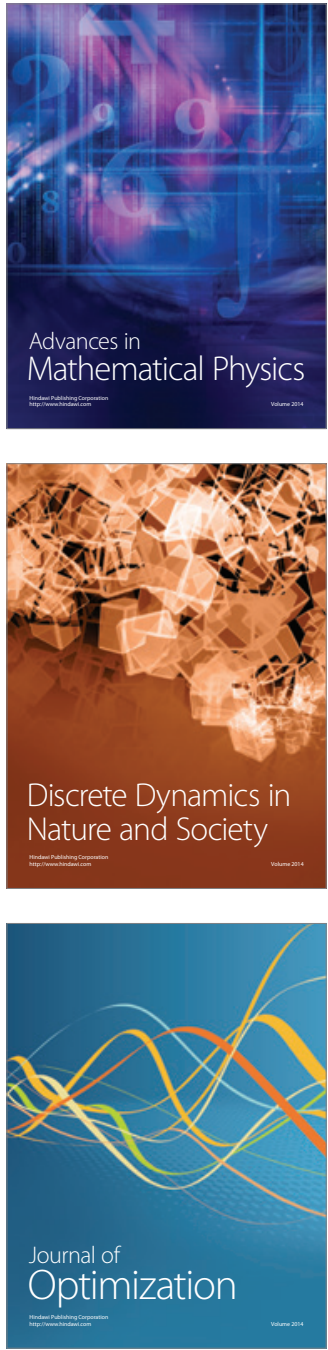